\title{
Assessment of Multidrug Resistance Tuberclosis Treatment Outcome in St. Petre's Tuberclosis Specialized Hospital, Addis Ababa, Ethiopia
}

\author{
Tsegaye Tulu, Mesfin Haile Kahissay
}

Department of Pharmaceutics and Social Pharmacy, School of Pharmacy, College of Health Sciences, Addis Ababa University, ETHIOPIA

\begin{abstract}
The purpose of this study is to assess the treatment outcome of MDR-TB among patients who are treated in St. Peter's TB Specialized Hospital from January 1, 2009 to December 31, 2010. A periodic retrospective cross sectional study was conducted on MDR-TB patient who were followed up to check their treatment out come. The study was utilized quantitative method for data collection by using patient registration book from MDR-TB clinic retrospectively. Using data collecting format, cure rate, failure rate, death rate, card number, sex, address, and comorbid illness was be collected. The association with the patient treatment outcome was assessed using SPSS version 15 for window using chi-square and odds ratio and Epi info for data entry. In this study the treatment outcome was measured by cure rate, death rate, failure rate and default rate. Base on this study that was done in St. Peter's TB specialized hospital from January 1, 2009 to December 31, 2010 cohort study, out of 166 MDR-TB patients, the treatment completion and cure rate was $7(4.2 \%)$. Regarding to sex more female $6(7.1 \%)$ of the treated patients were cured than male $1(1.23 \%)$ from the total females and males patients respectively. While $143(83.5 \%)$ were still on treatment, $1(0.6 \%)$ patient was defaulted from treatment for more than two months and the outcome was not traced. Treatment failure was not reported in this study. This finding indicates that from the study population, no treatment failure and one treatment interruption (default) was observed. In conclusion patients in this cross sectional study were found in good follow up and DOTS program. On the other hand the cure rate $7(4.2 \%)$ of this study showed that encouraging treatment outcome was obtained from the MDR-TB treatment plan. The result that was obtained from this study showed that the majority of MDR-TB patients in this retrospective study were found still on treatment.
\end{abstract}

Key words: Multidrug Resistance Tuberclosis Treatment, St. Petre's Tuberclosis Specialized Hospital, Ethiopia

\begin{tabular}{|c|c|}
\hline Source of Support: Nil, Conflict of Interes & t: None Declared \\
\hline $\begin{array}{l}\text { How to Cite: Tulu T and Kahissay MH. (2015) Assessment of Multidrug Resistance Tuberclosis Treatment Outcome in St. Pet } \\
\text { Specialized Hospital, Addis Ababa, Ethiopia. Malaysian Journal of Medical and Biological Research, 2(1), 7-18. }\end{array}$ & sis \\
\hline $\begin{array}{l}\text { This article is is licensed under a Creative Commons Attribution-NonCommercial } 4.0 \text { International License. } \\
\text { Attribution-NonCommercial (CC BY-NC) license lets others remix, tweak, and build upon work non-commercially, and although the new works must also } \\
\text { acknowledge \& be non-commercial. }\end{array}$ & (c) (i) \& \\
\hline
\end{tabular}

\section{INTRODUCTION}

Tuberculosis (TB) is still an infectious disease with high morbidity and mortality around the world. According to the World Health Organization (WHO), one-third of the population is infected and the estimated incidence of TB in 2002 was 8.8 million cases. Moreover, the worldwide emergence of multidrug resistant (MDR-TB) is a compounding problem as has been recently reported. Worldwide, the prevalence of primary and acquired MDR-TB among all cases of TB is $7.6 \%$ and $17.1 \%$, respectively (Haifeng Wang et al., 2008). Drug resistant (DR) and in particular multidrug-resistant (MDR-TB) defined as resistance to at least rifampitin and isoniazid (Juaeun N and Petms N, 2002).

Although progress has been made to reduce global incidence of drug-susceptible tuberculosis, the emergence of multidrug-resistant (MDR) and extensively drug-resistant (XDR) tuberculosis during the past decade threatens to undermine these advances. However, countries are responding far too slowly. Of the estimated 440, 000 cases of MDR tuberculosis that occurred in 2008, only $7 \%$ were identified and reported to WHO. Of these cases, only a fifth was treated according to WHO standards. Although treatment of MDR and XDR tuberculosis is possible with currently available diagnostic techniques and drugs, the treatment course is substantially more costly and laborious than for drug-susceptible tuberculosis, with higher rates of treatment failure and mortality (Neel G et al., 2010).

MDR-TB develops during treatment of fully-sensitive TB when the course of antibiotics is interrupted and the levels of drug in the body are insufficient to kill $100 \%$ of bacteria. This can happen for a number of reasons, Patients may feel better and halt their antibiotic course, drug supplies may run out or become scarce, or patients may forget to take their medication from time to time (www.cdc.gov/tb/publications/factsheets). In addition MDR-TB most commonly occur 
due to doctors giving in appropriate treatment or patients missing doses or failing to complete their treatment. MDR-TB strains are often less fit and less transmissible, and outbreaks occur more readily in people with weakened immune systems (e.g., patients with HIV) (CDC, 1991; Pitchenik AE et al., 1990; Fischl MA et al., 1992).

Nonetheless, a few countries provide examples of how existing technologies can be used to reverse the epidemic of MDR tuberculosis within a decade. Major improvements in laboratory capacity, infection control, and performance of tuberculosis control programs, and treatment regimens for both drug-susceptible and drug-resistant disease will be needed, together with a massive scale-up in diagnosis and treatment of MDR and XDR tuberculosis to prevent drugresistant strains from becoming the dominant form of tuberculosis (Neel G et al., 2010).

New diagnostic tests and drugs are likely to become available during the next few years and should accelerate control of MDR and XDR tuberculosis. Equally important, especially in the highest-burden countries of India, China, and Russia, will be a commitment to tuberculosis control including improvements in national policies and health systems that remove financial barriers to treatment, encourage rational drug use, and create the infrastructure necessary to manage MDR tuberculosis on a national scale. (Neel G et al, 2010).

The problem of drug resistant TB exists in different parts of Ethiopia, and data on patterns of resistance among Ethiopian isolates is ranging from $2 \%-21 \%$ for isoniazid, $2 \%-20 \%$ for streptomycin and $14 \%-15 \%$ for any of the drugs tested. MDRTB was also reported in about $1.2 \%$ of new cases and $12 \%$ of re-treatment cases. Little information is available in Ethiopia related to drug susceptibility assay on M. tuberculosis isolates from smear negative and culture positive sputum samples (Kassu Det al, 2008).

According to WHO 2008 report, in Ethiopia, 5858 MDR-TB cases (4964 among newly diagnosed and previously treated TB cases) were estimated to have occurred in 2006. The other survey that were conducted nationwide in 2005 (FMOH) on anti-TB drug resistance indicated that, among 804 newly diagnosed TB cases 1.6\% were found to be infected with MDRTB. The rate of MDR TB among specimens from 76 previously treated TB cases was $11.8 \%$ (FMOH, 2009).

\section{LITERATURE REVIEW}

The diagnosis of DR TB is still extremely low despite as many a half a million cases and 150,000 death from MDR-TB in 2008 globally according to WHO report 2008.in 2008 there were 29,432 MDR-TB cases reported throughout the world by 127 countries, but these cases represent only $7 \%$ of the MDR-TB cases estimated to have emerged at that year (WHO, 2010).The number of MDR-TB increases in Latvia and more than 200 patients began MDR-TB treatment each year (Leimane V and Leimans J, 2006).

Trends in drug resistance can be estimated for only 59 countries or sub national settings (eg, provinces) for which more than one drug-resistance survey was done between 1994 and 2009. Nonetheless, in six countries (Estonia, Hong Kong, Latvia, Russia [Orel and Tomsk oblasts], Singapore, and the USA) incidence of MDR tuberculosis is falling faster than is incidence of all forms of tuberculosis. In other countries, such as Lithuania, incidence of MDR tuberculosis is decreasing, but more slowly than the rate for all tuberculosis cases. In countries such as Peru, incidence of all tuberculosis cases is decreasing, but incidence of MDR tuberculosis is rising (Neel RG et al., 2010).

Most concerning is South Korea and Botswana, where all cases of tuberculosis are increasing in incidence and MDR tuberculosis is increasing even faster. Rising incidences of both tuberculosis and MDR tuberculosis in Botswana are being accelerated by high rates of HIV co-infection, which substantially increases progression to active disease after infection with M. bacterium tuberculosis. Furthermore, MDR tuberculosis and HIV co-infection has a high early mortality rate, especially in low-income settings Together with insufficient laboratory capacity for detection of such cases, this mortality could be masking innumerable local outbreaks of HIV associated MDR tuberculosis(Neel RG et al., 2010).

The study that was conducted on treatment outcome in India 2008 showed that, out of total 27 bacteriological proven cases of MDR-TB included in this study, 19 were males (mean age andweight 38.5 years and $52.6 \mathrm{kgs}$, respectively) and eight females (mean age and weight 34.3 years and $40.7 \mathrm{kgs}$, respectively). Amajority (18) was residents of Delhi and the rest hailed from different parts of North India. All of them had a history of previous treatment ranging from six to 34 months. Cavity on chest X-rays was seen in $81 \%$, while $44 \%$ showed extensive involvement. The patients received at least four "second line drugs" during their treatment with a mean of 6.2 antitubercular drugs during their intensive phase. Of the 27 patients, 13 were cured, 10 defaulted, one died, one is still on treatment and two were referred for surgery (Dhingra1V. K., 2008).

There was a study which was conducted in three TB Referral hospitals of Korea by Jeon DS, Shin DO, Park SK, et al in Dec 2010. 202 MDR-TB patients were included in the study, (37.1\%) had treatment success and (62.9\%) poor outcomes. Default rate was high $(37.1 \%$ ), comprising $59.1 \%$ of poor outcomes. The all cause mortality rate was $31.2 \%$ during the 3-4 year after treatment initiation. Finally, the author concluded that the treatment outcomes of patients with MDR-TB at the three tuberculoses hospitals are poor, which may reflect the current status of MDR-TB in the 
public sector of Korea. A more comprehensive program against MDR-TB needs to be integrated into the National Tuberculosis Program of Korea (Jeon DS et al., 2010).

The other study that was conducted on treatment outcome of MDR-TB by Ferrer G et al, 2010 in Texas, out of bacteriological proven 45 MDR-TB patients, among which 30 were cured, 3 showed treatment failure, 3 died, and 10 was abandoned treatment in the study period. The author concluded that successful outcome of MDR-TB was shown during the study period (Ferrer G et al, 2010).

Study done on clinical outcome of MDR-TB in Latvia showed that, of the 204 patients assessed, 55 (27\%) had been newly diagnosed with MDR-TB, and 149 (73\%) had earlier been treated with first-line or second-line drugs for this disease. Assessment of treatment outcomes showed that $135(66 \%)$ patients were cured or completed therapy, 14 (7\%) died, 26 (13\%) defaulted, and treatment failed in 29 (14\%). Of the 178 adherent patients, 135 (76\%) achieved cure or treatment completion (Vaira L et al, 2005)

A retrospective study was conducted on outcome of hospitalized MDR-TB patients in Israel national referral tuberculosis centers in 2010. One hundred thirty-two patients were identified with a median age of 40 years and male predominance $(77 \%)$. The majority of the patients were immigrants from FSU (83\%) and Ethiopia (7.6\%). They were characterized by alcohol (25.8\%) and IV drug abuse (23.5\%), presented with advanced disease manifested by hypoalbuminemia (50.8\%) and smear positivity (70.5\%). Cure was achieved in 50.3\% and 30.4\% died. Finally, the authors recommended that there should be focused on earlier diagnosis and treatment in a well controlled hospital environment and to professional support groups to attend to this population's special needs (Bendayan D et al, 2011).

The study that was done on outcome of MDR-TB in Taipei 1992-1996 cohort study, with 6-year follow-up, showed that among the 153 patients who were cured, full information was available to evaluate the entire bacteriological course. The study showed that (90.8\%-82.7\%) had sputum culture conversion within 3months, and (90.6\%) had sputum culture conversion within 6 months. of the 29 patients who failed $(90.0 \%)$ remained persistently positive up to 12 months. In conclusion, Patients who need modification of MDR-TB regimen due to the consideration of inadequate regimen should be classified as failed and re-registered as retreatment cases (Chiang C-Y et al., 2006).

Africa experienced an estimated 69,000 cases of MDR-TB in 2008.Since Africa countries have the highest incidence of TB per population in the world, even at low level of drug resistance the case load of MDR-TB patients become very high. As a result the rate of MDR-TB cases arising per 100,000 population and in some southern Africa countries the burden of MDR-TB are 5 to 6 times higher than those of china and India. In addition treating MDR-TB can cost 50 and 200 times more than first line treatment for non resistance cases (WHO, 2010).

Despite the increase in HIV-associated tuberculosis seen in most African countries, an upsurge of MDR-TB has not been documented. Exceptions include Mozambique and Côte d'Ivoire, where more than 3\% of new cases are MDR$\mathrm{TB}$ and in Yaonde, Cameroon, where the corresponding figure rises to $27 \cdot 6 \%$ (Alimuddin Z and John M, 2001).

A study was done on treatment outcome in Cameroon 2002 to see the treatment outcomes in 410 patients with drugsusceptible tuberculosis (DS-TB) and 150 patients with drug-resistant tuberculosis (DR-TB) among a total of 560 adult patients ( $\geq 15$ years old) notified with smear-positive pulmonary tuberculosis between July 1997 and June 1998 in the West Province of Cameroon and treated with World Health Organization (WHO) standard regimens under field conditions. Out of the 410 patients 332(81\%) with DS-TB were cured, compared to $109 / 150$ (72.7\%) patients with DR-TB. Seven patients (1.7\%) failed treatment in the DS-TB group vs. $9(6.0 \%)$ in the DR-TB group.This study recommend that to prevent development of drug-resistance, the proportion of defaulters must be decreased and prevention and control strategies endorsed by the WHO and the International Union Against Tuberculosis and Lung Disease must be implemented nation-wide (Juaeun N and Petms N, 2002).

Study on drug resistant tuberculosis in sub-Saharan Africa showed that MDR, i.e. resistance to at least rifampiein (Rmp) and isoniazid (INH), was observed in (1.3\%) out of 236 new cases and in (6.5\%) out of 62 retreated cases. For new cases, single drug resistance to INH, RMP and ethambu- tol (E) was 3\%, $0.4 \%$ and $4.2 \%$ respectively; for retreated cases it was $14.5 \%, 1.6 \%$ and $6.5 \%$ respectively. Based on the estimate of the size of the TB problem in subSaharan Africa by the year 2000 (Schulzer), the treatment outcome calculated that the region should expect between 15, 543 and 223,417 cases of MDR, all forms combined (between 2.3 and 32.7 per 100,000 inhabitants), by the end of the century. Finally the author's data urgently point to the need for drug resistance surveys, followed by continuous drug resistance monitoring in high TB prevalence areas(Carpels $G$ et al., 1995).

Multidrug-resistant tuberculosis is defined as infection caused by Mycobacterium tuberculosis resistant to isoniazid and rifampin. Extensively drug-resistant TB was defined initially as infection causedby M. tuberculosis not only resistant to isoniazid and rifampin but also to at least 3 of the 6 classes of second-lineagents approved for the treatment of TB (amino glycosides, polypeptides, fluoroquinolones, thioamides, cycloserine, and para- 
aminosalicyclic acid).The WHO Global Task Force on XDR-TB modified the definition in October 2006 following the report of the outbreak in KwaZulu Natal.Because drug susceptibility testing is reliable only for amikacin, kanamycin, capreomycin, and fluoroquinolones among the second-line agents, the definition of extensively drugresistant TB is now resistance to isoniazid and rifampin in addition to resistance to any fluoroquinolone and any of the second-line injectable drugs: amikacin, kanamycin, and capreomycin (Miguel G, 2008).

The first detailed global prevalence data on XDR-TB came to light via extended drug sensitivity testing (DST) of a convenience sample of supranational TB reference laboratory isolates collected between 2000 and 2004. Eighteen thousand individual isolates were tested, of which more than $3500(19.9 \%)$ were MDR, and of these resistant strains, $7 \%$ fulfilled the laboratory definition of XDR-TB. Geographical spread was very uneven. As a proportion of MDRTB isolates, the levels were highest at around 15\% in South Korea and Eastern Europe (Western Asia), whereas of 156 MDR isolates from Africa (Middle East), just one was XDR-TB (CDC, 2006).

While suffering with a very high and expanding TB caseload, proportional XDR prevalence among MDR isolates in sub-Saharan Africa appears to be low, just as the proportion of MDR strains is small compared with the drugsensitive TB burden. However, 2006 saw dramatic reports concerning a potential XDR-TB outbreak in Tugela Ferry, a small town in the KwaZulu-Natal province of South Africa (Gandhi NR et al., 2006).

The initial identification of a number of cases of XDR-TB among treatment non-responsive subjects prompted the institution of extended SLD sensitivity testing for all patients presenting with signs or symptoms of TB. In this group, 475 of 1428 patients had culture-positive TB, of whom 39\% had MDR strains, and of these MDR isolates, $16 \%$ (30) were XDR: a total of $6 \%$ of all (unselected) TB cases. The case fatality rate across all 53 of the Tugela Ferry XDRTB patients was appallingly high: 52 died, with a median survival time after sputum sample of just 16 . Subsequent reports have described further expansion of the XDR-TB caseload in South Africa, with more than 250 cases in total (Kapp C, 2006).

The treatment regimens were variable and consisted of five drugs in the intensive phase which included any firstline anti-TB drug to which the isolate was still susceptible, an amino glycoside or capreomycin, a fluoroquinolone (ofloxacin, ciprofloxacin or levofloxacin), any of the second-line drugs including prothionamide or ethionamide, cycloserine, PAS, or clarithromycin (Tupasi T.E, 2003).

The intensive phase was given under directly observed treatment (DOT) in the clinic daily until the patient attained six consecutive monthly negative sputum cultures. Thereafter, the injectible agent was discontinued and the patient was given continuation therapy with the remaining four drugs until they attain 18 months of consecutively negative sputum cultures. During the continuation phase, the patients were given medications on DOTS twice or thrice weekly. In-between clinic visits, they took the medications at home by self-administered therapy (SAT) supervised by their treatment partners. In several occasions, the patients' medications were modified due to adverse drug events (TupasiT.E, 2003).

\section{SigNIFICANCE OF THE STUDY}

Despite the invention of effective drug for the treatment of TB before a long period and the introduction of DOTS, TB still become a global problem especially in resource limited countries including Ethiopia. Tuberculosis is one of the most resource dependent diseases in the world and affects the most economical productive age group especially those population live with HIV AIDS. MDR-TB is one of the challenges that exacerbate the DOTS program and successful treatment outcome of different countries in the world especially in developing countries like Ethiopia.

In Ethiopia there was no much effort done regarding to the patient adherence, incomplete and inadequate treatment and identification of the patient's diagnosis these factors leads to the high prevalence of MDR-TB cases in different part of the country. In Ethiopia treatment of MDR-TB,was started in February, 2009 by FMOH with collaboration of GLC (green light committee)and other funding NGOS in St. Peter's TB specialized hospital.

Due to the late establishment of treatment strategy and long duration of treatment course there is no study that shows the treatment outcome of MDR-TB. Most probably this study is the first documented study on treatment outcome of MDR-TB so that it is really help to know the status of the patient outcome in the county. In addition to this, this study serves as the baseline study for future assessment of the patient treatment outcome of MDR-TB. Since the burden of MDR-TB in the country is increasing from time to time, this study will give information to the policy maker about the situation of MDR-TB treatment out come to the country. 


\section{OBJECTIVE}

General Objective of the present study is to assess the treatment outcome of MDR -TB treatment among patients who are treated in St. Peter's TB Specialized Hospital from January 1, 2009 to December 31, 2010. The specific objectives are:

- To determine cured rate of MDR-TB among MDR-TB patients treated.

- To determine failure rate of MDR-TB among MDR - TB Treated patients.

- To determine death rate of MDR-TB among MDR-TB Treated patients.

- To determine treatment default rate of MDR-TB among MDR-TB patient treated.

\section{Methodology}

A retrospective cohort study was conducted on MDR-TB patient who were longitudinally followed to check their treatment out come. The study was utilized quantitative method for data collection by using patient registration book from MDR-TB clinic retrospectively. Using data collecting format, cure rate, failure rate, death rate, card number, sex, address, and co-morbid illness (HIV status) was collected. The association with the patient treatment outcome was assessed using SPSS version 16 for window using chi-square and odds ratio and Epi info for data entry.

St. Peter's TB Specialized Hospital is a governmental Hospital under Federal Ministry of Health of Ethiopia. The hospital provides various services especially in Tuberculosis diagnosis and Treatment and additionally Internal medicine, Pediatrics, Mothers and Child health, antiretroviral therapy, voluntary counseling and Testing, Laboratory, Regular and Special pharmacy, admission of 250 beds capacity are the major services of the hospital. Based on unpublished human resource manual, 2007/8 the hospital laboratory was well furnished and equipped with different instruments. Currently; the hospital has a responsibility to serve the people of Ethiopia by providing the second line anti TB drugs in the country. The MDR-TB ward was renovated and started inpatient service in 2009 with 45 bed capacity.

Study was conducted from January 1, 2009 to December 31, 2010 on MDR-TB patients registered for treatment in St. Peter's TB Specialized hospital.

\section{Population and Sample}

All tuberculosis patients who were registered for treatment of both active and multidrug resistance tuberculosis at St. Peter's TB Specialized Hospital from January 1, 2009 to December 31, 2010.

All cases of MDR-TB patients who were resisted and started treatment in the time period between January 1, 2009 and December 31, 2010 who have been on DOTS regimen of MDR-TB drug at St, peter's TB specialized Hospital.

For quantitative method MDR-TB patients registered between January 1, 2009 and December 31, 2010 in St. peter's TB Specialized Hospital were taken and some socio demographic characteristics of MDR-TB patient (age, sex, and address), treatment outcome (cure rate, failure rate, default rate and death rate), treatment period (intensive and continues phase) co-morbid illness (HIV status of the patients) was collected from patient registration log using two pharmacist who were trained on the data collection process and who have previous experience of MDR-TB drug dispensing.

\section{Study Variable}

Dependent Variable:

- Cured rate

- Failure rate

- Death rate

- Treatment default rate

Independent Variable:

- Age

- Sex

- HIV status

- Address

- Duration of treatment 


\section{Data collection}

The data was extracted from treatment charts by using standardized checklist, and obtained baseline demographic and risk-factor information, including age, sex, address, and duration of treatment and HIV status of the patients. Also the treatment outcome of the patients (cure rate, death rate, default rate and failure rate) was collected from St. Peter's TB specialized hospital MDR-TB case team patient registration book.

Epi-info version 6.04 and statistical package for social science (SPSS) version 16.0 programs was used for quantitative data entry and analysis respectively. All the data that were collected was checked for accuracy and completeness prior to entry into the data base. After the data entry, the data base information was cross checked with the data collection forms before commencement of analysis.

\section{RESULT}

The patient registration book was reviewed for patients enrolled from January 1, 2009 to December 31, 2010.A total of 340 cultures positive screened MDR-TB patients registered for treatment in St. Peter's TB Specialized hospital during this period. Among these patients $166(48.8 \%)$ of them were started treatment in the study period and included in the study.

5.1. Socio-demographic characteristics of MDR-TB patients who were treated at St. Peter's RB Specialized hospital in the study period.

Table 1 shows the socio demographic distribution of MDR-TB patients enrolled in the study. From the 166 cases included in the study (mean age 9.75 years), $147(88.6 \%$ ) were in the most productive age group (i.e. between $15-45$ years). Most of the MDR cases occurred in the age group of 25-34 (39.75\%), followed by 15-24 (31.13\%) with mean age of 29.89 years (range, 9-75 years). With regard to geographical distribution, the majority, 134(80.7\%), were from Addis Ababa followed by Oromiya region13 (7.8\%) near to Addis Ababa.

The majority of cases were females 85 (51.2\%). Males represented $81(48.8 \%)$ of the population (Table 1$)$. The female to male ratio was 1.04:1, the ratio shows that almost both female and male affect equally. Most of the MDR cases occurred in the age group of 25-34 (39.75\%), followed by 15-24 (31.13\%). The mean age of the study population was 29.89(range, 9-75 years). In case of female and male, the ratio was higher in the younger population (under age of 34 years, $77.6 \%$, and $67.9 \%$ respectively) than in the elder population (over age of $35,22.35 \%$ and $32.1 \%$ respectively). In the case of males over age of 35 the ratio was 1.4:1 with that of female. The majority of the study population $85(52.2 \%)$ were found in the continuation phase than the intensive phase $81(48.8 \%)$.

Table 1: Socio demographic distribution of MDR-TB patients in St. peter's TB specialized hospital from January 1, 2009 to December 31, 2010(N=166)

\begin{tabular}{|c|c|c|}
\hline & Frequency & Percentage \\
\hline $0-14$ & 3 & 1.8 \\
\hline $15-24$ & 52 & 31.3 \\
\hline $25-34$ & 66 & 39.7 \\
\hline Age group $\quad 35-44$ & 29 & 17.4 \\
\hline $45-54$ & 10 & 6.0 \\
\hline $55-64$ & 4 & 2.4 \\
\hline$>65$ & 2 & 1.2 \\
\hline Total & 166 & 100 \\
\hline Gender Female & 85 & 51.2 \\
\hline Male & 81 & 48.8 \\
\hline Total & 166 & 100 \\
\hline Addis Ababa & 134 & 80.7 \\
\hline Oromiya & 13 & 7.8 \\
\hline SNNP & 8 & 4.8 \\
\hline Address Tigray & 5 & 3.0 \\
\hline Others & 6 & 3.7 \\
\hline Total & 166 & 100 \\
\hline Treatment duration & 81 & 48.8 \\
\hline Continues & 85 & 52.2 \\
\hline Total & 166 & 100 \\
\hline
\end{tabular}

Others: Amahara, Benishangul and Gambela 
Distribution of MDR-TB patients by HIV status, age group, gender and address in St. Peter's TB Specialized hospital in the study period

Out of the study population 40(24.1\%) were HIV positive, $115(69.3 \%)$ were HIV negative and $11(6.6 \%)$ were unknown status. Among the HIV positive group, 23(57.5\%) were females and 17(42.5\%) were males indicating that HIV sero-positivity rate among MDR TB patients was higher among females than males. With regard to HIV status among the different age groups, the age group between $25-34$ years, $13(19.7 \%)$ and between $35-44$ years, $12(19.7 \%)$ were constituting the highest proportion of patients that were affected by both MDR-TB and HIV. Among the 134 patients who came from Addis Ababa, 33(24.6\%) of them were HIV positive and the rest were from other regions of the country.

The majority of the study population which was affected by MDR-TB was HIV negative with total number of 115(69.3\%).Among HIV negative patients55(47.8\%) of them were females and 60(52.2\%) males. The rest $11(6.6 \%)$ of the patients were not tested for HIV, but the proportion of HIV positive patients may be different from what have been shown on the table 2. if all MDR-TB patients were willing to be tested for HIV. The association of the other data was shown in table 2.

Table 2: Distribution of MDR-TB patients by HIV status, age group, gender and address in St. peter's TB specialized hospital from January 1,2009 to December 31,2010(N=166)

\begin{tabular}{|l|l|l|l|l|l|}
\hline & $\begin{array}{l}\text { Positive } \\
\mathrm{N}(\%)\end{array}$ & $\begin{array}{l}\text { Negative } \\
\mathrm{N}(\%)\end{array}$ & $\begin{array}{l}\text { Unknown } \\
\mathrm{N}(\%)\end{array}$ & Total N & p-value \\
\hline $\mathbf{0 - 1 4}$ & $3(100.0)$ & 0.00 & 0.00 & 3 & \\
\hline $\mathbf{1 5 - 2 4}$ & $7(13.5)$ & $43(82.7)$ & $2(3.8)$ & 52 & \\
\hline $\mathbf{2 5 - 3 4}$ & $13(19.7)$ & $47(71.2)$ & $6(9.1)$ & 66 & \\
\hline Age group 35-44 & $12(41.4)$ & $15(51.7$ & $2(6.9)$ & 29 & $\mathrm{P}=0.269$ \\
\hline $\mathbf{4 5 - 5 4}$ & $4(40.0)$ & $6(60.0)$ & 0.00 & 10 & \\
\hline $\mathbf{5 5 - 6 4}$ & $1(25.0)$ & $2(50.0)$ & $1(25.0)$ & 4 & \\
\hline$>\mathbf{6 5}$ & & $2(100.0)$ & & 2 & \\
\hline Total & $40(24.1)$ & $115(69.3)$ & $11(6.6)$ & 166 & \\
\hline Gender & & & & & \\
\hline Female & $23(27.1)$ & $55(64.7)$ & $7(8.2)$ & 85 & $\mathrm{P}=0.398$ \\
\hline Male & $17(21.0)$ & $60(74.1)$ & $4(4.9)$ & 81 & \\
\hline Total & $40(24.1)$ & $115(69.3)$ & $11(6.6)$ & 166 & \\
\hline & & & & & \\
\hline Addis Ababa & $33(24.6)$ & $92(68.7)$ & $9(6.7)$ & 134 & \\
\hline Oromiya & $2(15.4)$ & $10(76.9)$ & $1(7.7)$ & 13 & \\
\hline Address SNNP & $3(37.5)$ & $4(50.0)$ & $1(12.5)$ & 8 & $\mathrm{P}=0.935$ \\
\hline Tigray & $2(40.0)$ & $3(60.0)$ & & 5 & \\
\hline Others & 0.00 & $6(100.0)$ & 0.00 & 6 & \\
\hline Total & $40(24.1)$ & $115(69.3)$ & $11(6.6)$ & 166 & \\
\hline
\end{tabular}

HIV Status

Others: Amahra, Gabela and Benishangul chi-square

Distribution of treatment outcome by HIV status, age, gender and treatment duration among MDRTB patients at St. Peter's TB Specialized hospital in the study period.

In this study the treatment outcome was measured by cure rate, death rate, failure rate and default rate. The cure rate and treatment completion was considered as one variable due to consideration of similar outcome in the registration book. Out of $166 \mathrm{MDR}-\mathrm{TB}$ patients, the treatment completion and cure rate was $7(4.2 \%)$. While $143(83.5 \%)$ were still on treatment, $1(0.6 \%)$ patient defaulted from treatment follow-up for more than two months. Treatment failure was not reported in this study. With regard to gender, more females $6(7.1 \%)$ were cured as compared to males $1(1.23 \%)$.

There is no cure rate difference between HIV positive and HIV negative patients from the total population of both HIV+ and HIV- patients $3(7.5 \%)$ and $3(2.6 \%)$ respectively. From the total (115) HIV negative patients $11(9.6 \%)$ died during the treatment course as compared with $2(5.0 \%)$ HIV positive patients and the rest $2(18.2 \%)$ were patients with unknown status.

Among MDR-TB patients who were started the treatment in the study period, $15(9.1 \%)$ of them died during the treatment course and among these patients $12(80.0 \%)$ died in the first two months of the intensive phase and the rest $3(20.0 \%)$ died after more than six culture conversion during continuation phase. More deaths occurred in the productive age group than in the elderly. The association of the other data was shown in table 3. 
Table 3: Distribution of treatment outcome by HIV status, age, gender and treatment duration among MDR-TB patient in St. peter's TB specialized hospital from January 1,2009 to December 31,2010 (N=166). Treatment outcome

\begin{tabular}{|l|c|c|c|c|c|c|}
\hline & $\begin{array}{c}\text { Cured } \\
\text { Frequency } \\
\mathbf{( \% )}\end{array}$ & $\begin{array}{c}\text { Defaulted } \\
\text { Frequency } \\
\mathbf{( \% )}\end{array}$ & $\begin{array}{c}\text { Died } \\
\text { Frequency } \\
\mathbf{( \% )}\end{array}$ & $\begin{array}{c}\text { On treatment } \\
\text { Frequency } \\
\mathbf{( \% )}\end{array}$ & Total & P-value \\
\hline $\mathbf{0 - 1 4}$ & 0.00 & 0.00 & 0.00 & $3(100.0)$ & 3 & \\
\hline $\mathbf{1 5 - 2 4}$ & $1(1.9)$ & 0.00 & $5(9.6)$ & $46(88.5)$ & 52 & \\
\hline Age group 25-34 & $3(4.5)$ & 0.00 & $5(7.6)$ & $58(87.9)$ & 66 & \\
\hline $\mathbf{3 5 - 4 4}$ & $1(3.4)$ & 0.00 & $3(10.3)$ & $25(86.2)$ & 29 & \\
\hline $\mathbf{4 5 - 5 4}$ & $2(20.0)$ & 0.00 & $2(20.0)$ & $6(60.0)$ & 10 & $\mathrm{P}<0.001$ \\
\hline $\mathbf{5 5 - 6 4}$ & 0.00 & $1(25.0)$ & 0.00 & $3(75.0)$ & 4 & \\
\hline >65 & 0.00 & 0.00 & 0.00 & $2(100.0)$ & 2 & \\
\hline Total & $7(4.2)$ & $1(0.6)$ & $15(9.1)$ & $143(86.1)$ & 166 & \\
\hline & & & & & & \\
\hline Gender female & $6(7.1)$ & 0.00 & $8(9.4)$ & $71(83.5)$ & 85 & \\
\hline Male & $1(1.23)$ & $1(1.23)$ & $7(8.6)$ & $72(88.8)$ & 81 & $\mathrm{P}=0.208$ \\
\hline Total & $7(4.2)$ & $1(0.6)$ & $15(9.1)$ & $143(86.1)$ & 166 & \\
\hline & & & & & & \\
\hline HIVstatus positive & $3(7.5)$ & $1(2.5)$ & $2(5.0)$ & $34(85.0)$ & 40 & $\mathrm{P}=0.273$ \\
\hline Negative & $3(2.6)$ & 0.00 & $11(9.6)$ & $101(87.8)$ & 115 & \\
\hline unknown & $1(9.1)$ & 0.00 & $2(18.2)$ & $8(72.7)$ & 11 & \\
\hline Total & $7(4.2)$ & $1(0.6)$ & $15(9.1)$ & $143(86.1)$ & 166 & \\
\hline & & & & & & \\
\hline DurationofRx Intensive & 0.00 & $1(1.2)$ & $12(14.8)$ & $68(84.0)$ & 81 & $\mathrm{P}=0.003$ \\
\hline Continues & $7(8.4)$ & 0.00 & $3(3.6)$ & $75(88.0)$ & 85 & \\
\hline Total & $7(4.2)$ & $1(0.6)$ & $15(9.1)$ & $143(86.1)$ & 166 & \\
\hline & & & & & & \\
\hline Address Addis Ababa & $6(4.6)$ & $1(0.6)$ & $12(9.0)$ & $115(85.8)$ & 134 & \\
\hline Oromiya & 0.00 & 0.00 & $1(7.7)$ & $12(93.3)$ & 13 & $\mathrm{P}=0.836$ \\
\hline SNNP & $1(12.5)$ & 0.00 & 0.00 & $7(87.5)$ & 8 & \\
\hline Tigray & 0.00 & 0.00 & 0.00 & $5(100.0)$ & 5 & \\
\hline Others & 0.00 & 0.00 & $2(33.3)$ & $4(6.7)$ & 6 & \\
\hline Total & $7(4.2)$ & $1(0.6)$ & $15(9.1)$ & $143(86.1)$ & 166 & \\
\hline & & & & & \\
\hline & & & & & & \\
\hline
\end{tabular}

Others:Amahra, Bambela, Benishagul Chi-square

\section{Distribution of defaulters and deaths verses the phase of treatment among MDR-TB patients.}

In this study only one patient defaulted in the intensive phase of the treatment course in the study period and the final outcome could not be traced. Most of the deaths, $12(80.0 \%)$ occurred in the intensive phase than the continuation phase $3(20.0 \%)$.

Table 4: Distribution of defaulter and death among MDR-TB patients in the defaulting period in St. Peter's TB Specialized hospital from January 1, 2009 to December 31, 2010. Duration of treatment

\begin{tabular}{|l|r|r|r|}
\hline & Intensive phase (\%) & Continues phase (\%) & Total \\
\hline Defaulter & $1(100.0)$ & 0.00 & 1 \\
\hline Death & $12(80.0)$ & $3(20.0)$ & 15 \\
\hline
\end{tabular}

\section{Discussion}

According to the finding treatment outcome was shown as, cure $7(4.2 \%)$, death $15(9.6 \%)$, default $1(0.6 \%)$, still on treatment $143(86.1 \%)$ and no treatment failure was observed in the study period. This result may not give the exact figure of treatment outcome because most of the patients in this cohort study were found still on treatment.

As observed from the result MDR-TB was highly affecting the most productive age group (88.6\%) with mean age of 29.89 years which almost coincide with some finding and truth of the world (FMOH, 2008). HIV status of the patients were not associated with the outcome of MDR-TB with the $\mathrm{p}=0.266$. According to this finding more females are affected by MDR-TB than males $(51.2 \%)$ and $(48.8 \%)$ respectively, but this difference is not statistically significant with a $\mathrm{p}$-value $=0.396$. The probable reason for this result may be females have more contact with the patients during home care in the family and the other probable reason may be due to high blood flow during menstruation and delivery that may lead to compromising the immunity. Cure rate were higher in females $6(7.1 \%)$ than in males (table 3). These findings are consistent with the results of other studies in sub-Saharan Africa (GlynnJ et al., 1998; Lienhardt, Cetal., 1998). 
In this finding most of the independent variable have no association with the treatment outcome except age and treatment duration with the $\mathrm{p}<0.001$ and $\mathrm{p}=0.032$ respectively. Tuberculosis is the most common opportunistic infection and cause of death among HIV infected patients in resource-limited settings (De Cock KM et al 1992, WHO, 2003). Patients with HIV infections are particularly vulnerable to primary disease following infection with tuberculosis, (Neel R, 2010) and therefore are at high risk of illness and mortality when exposed to drug-resistant tuberculosis strains. Different studies done on the treatment outcome of MDR-TB found that the HIV status of the study population directly associated with the outcome but in this study the association is not statistically significant with the $p=0.266 \mathrm{so}$, this finding is important for the design of policy guidelines and program for management, reduction of stigma associated with a fatal prognosis, and advocacy purposes and this result is similar with study that done in south Africa KwaZulu Natal in 2006 and 2009( Gandhi NR, 2006).

Since the study was conducted using only patient registration book, some of the factors that may associate with both the successful outcome and poor outcome like (socio-economic status, types of MDR-TB like acquired and new MDR-TB and side effect of the drug) were not included (FMOH, 2009) So more investigation is essential to have a full information and finding on treatment outcome of MDR-TB.

The burden of death due to MDR-TB in the world is high. The death rate of this study was $9.1 \%$ which was relatively lower than study done on treatment outcome of hospitalized MDR-TB patients in Israel national referral tuberculosis centers in 2010 (Bendayan D et al, 2011). This is result may not be the actual death rate because the study was conducted based on patient registration book and there is problem of tracing patients who were interrupted treatment in the treatment duration. In this study more death occurs in the intensive phase of the treatment duration $12(14.8 \%)$ than in continuation phase $3(3.6 \%)$. The probable reason may be most of the patients start treatment after get wreaked and the side effect of the drug also exacerbate the situation. The death rate may be decreased if the patient managing system will be setup (FMOH, 2010). Other observation from this result showed that HIV was not the main cause of death of the patients, 2(5.0\%) HIV positive and 11(9.6\%) HIV negative respectively this result coincides with study done on treatment outcome of MDR-TB in South Africa KwaZulu Natal (Gandhi NR, 2006).

In this study there is no treatment failure out of 166 study subjects enrolled in the study from patient registration book but one patient was complete 24 month full treatment duration and still on treatment with 28 month duration. This is due to the lung damage of the patient and waits for lung surgery (Jeon DS et al., 2010).

The default rate of this study was $0.6 \%$, which is relatively lower than defaulting rate done in three TB Referral hospitals of Korea (Jeon DS et al., 2010). This shows that most of the patients in the study period were in the strategy of DOTS program sated by WHO and there was a restrict follow up of the patients by the physician and nurses. This patient interrupted the medication in the intensive phase of the treatment duration. The probable reason for the interruption may be the side effect of the drug itself (Hopewell C.H and Chisson R.E, 2000).

Most of the patients $(88.0 \%)$ of this study were found in continues phase of the treatment duration. The most probable reason may be the early establishment of the treatment strategy by ministry of health with collaboration of other NGOS like GHC (global health committee), GLC (green light committee) and TB CAP in St. Peter's TB Specialized hospital. Based on the selection criteria and patients condition, in the first round 8 patients were started treatment, in the second round about 18-20 patients were started treatment and followed by 45 patients (FMOH, 2008).

About $(80.7 \%)$ of the study population who were start treatment came from Addis Ababa. This result couldn't show the exact figure of Addis Ababa prevalence because St. Peter's TB specialized hospital is the only setting that the treatment of MDR-TB first started so that most of the population in the study came from different parts of the country to Addis Ababa in order to get treatment (FMOH,2008).

\section{LIMITATION OF THE STUdY}

Although this study produced some significant findings, the generalization of the study results is subject to the following limitations: the first limitation was since, the study was conducted by using the secondary data from the $\log$ book and restricted to retrospective study; it is difficult to assess the level of data quality in the hospital. The second limitation of this study was St. Peter's TB specialized hospital is the only facility for MDR-TB treatment in the country in this study period so that patients from different part of the country came to Addis Ababa in order to get treatment therefore the result obtained from this study couldn't show the exact figure of Addis Ababa's prevalence of MDR-TB situation even the other region of the country. The third limitation was it is difficult to extract independent variable like socio-economic status, risk factors for poor outcome types of MDR-TB and resistance pattern of the second line drugs from patient registration book so that this study was not exactly evaluate the treatment outcome of the patients. 


\section{CONCLUSIONS}

The main purpose of this study is to evaluate the treatment outcome of MDR-TB patients and to asses some factor contributing values to successful and poor treatment outcome. The important area that considered as good treatment outcome were high cure rate, low death rate, low default rate and low failure rate. The result that was obtained from this study shows that the majority of MDR-TB patients in this retrospective study were found on treatment. This result may differ when all patients still on treatment will complete their treatment duration. From quantitative analysis the death rate of this observation relatively high and the main factor associated with this result may be the side effect of the drug and long duration of treatment.

Based on the finding HIV related MDR-TB was uncommon in Ethiopia. This finding is important for the design of policy guidelines and program for management, reduction of stigma associated with a fatal prognosis, and advocacy purposes.

Even though the treatment outcome of this study is encouraging the study indicates that successful treatment outcome of MDR-TB is a challenging and need effort and support from the concerned body so that linking MDR-TB outcomes with data from other surveillance systems markedly improved the mortality estimates and allowed us to detect recurrent case of the disease. MDR-TB reflects the poor primary management of the disease. It is mainly caused by the failure to ensure compliance, rather than the failure of the drugs to cure. The highest priority in fighting MDR-TB therefore must be its prevention.

\section{RECOMMENDATION}

- Relatively the death rate of this study is high so that patient care at the start of treatment specially the first two months of the intensive phase should be managed properly

- There should be fully organized facilities for treatment and management of MDR-TB patients in different parts of the countries

- Managing the contacts of MDR-TB patients, provision of social support to the patients and their families during treatment

- Provide education to the patient and patient's family with the goal of successful completion of therapy

- Provision of services to manage the anticipated frequent, and sometime severe, adverse drug reactions

- Provision of daily DOT over an extended period of 24-27 months; ensuring uninterrupted supply of DOTS Plus drugs

- Ensuring the establishment of long term sustainable DOTS Plus services under the NTCP.

- Staff training, motivation, and supervision

- Improve drug supply management chain for MDR-TB

- Provide access to comprehensive primary care

- Provision of health education on MDR-TB transmission to the public

\section{REFERENCES}

Asebe G, Ameni G and Tafess K. 2014. Ten years tuberculosis trend in Gambella Regional Hospital, South Western Ethiopia. Malaysian Journal of Medical and Biological Research, 1, 18-24.

Bendayan D, Hendler A, Polansky V (2011). Outcome of hospitalized MDR-TB patients: Israel 2000-2005. Eur J Clin Microbiol Infect Dis. Mar, 30(3):375-9. Epub, 2010 Oct 23.

Carpels G, Fissette K., Limbana V, Van Deun A (1995). Drug resistant tuberculosis in sub-Saharan Africa an estimation of incidence and cost for the year 2000.Tubercle and Lung Disease, 76, 480-86).

Centers for Disease Control (1990). Outbreak of multidrug-resistant tuberculosis-Texas, California, and Pennsylvania. MMWR Morb Mortal Wkly Rep, 39 (22): 369-72).

Centers for Disease Control (1991). Nosocomial transmission of multidrug-resistant tuberculosis among HIV-infected personsFlorida and New York, 1988-1991. MMWR Morb Mortal Wkly Rep 40: 585-591.

Centers for Disease Control (1991). Transmission of multidrug-resistant tuberculosis from an HIV-positive client in a residential substance-abuse treatment facility-Michigan. MMWR Morb Mortal Wkly Rep, 40: 129-131.

Centers for Disease Control Prevention (2006). Emergence of Mycobacterium tuberculosis with extensive resistance to second-line drugs-worldwide, 2000-2004. Morb. Mortal. Wkly Rep. 55, 301-305.

Chan E D and Iseman M D (2002). Current medical treatment for tuberculosis; Br. Med. J. 325 1282-1286.

Chiang C-Y,Eur respire J (2006); 28:980-985.

Datta M, Radhamani M P, Selvaraj R, Paramasivan C N, Gopalan B N, Sudeendra C R and prabhakar R (1993). Critical assessment of smear-positive pulmonary tuberculosis patients after chemotherapy under the district tuberculosis programme; Tuber. Lung Dis. 74 180-186.

De Cock KM, Soro B, Coulibaly IM, Lucas SB (1992). Tuberculosis and HIV infection in sub-Saharan Africa. JAMA 1992; 268: 1581-87. 37 
Dhingra V. K, Rajpal S, Anshu Mittal and Hanif M. (2008). Outcome of MDR-TB cases treated by individualized regimens at a tertiary level clinic. Indian J Tuberc, 55:15-21.

Drobniewski F A and Balabanova Y M (2002). The diagnosis and management of multiple-drug- resistant tuberculosis at the beginning of the new millennium; Int. J. Infect. Dis. 6 S21-S31.

Edlin BR, Tokars JI, Grieco (1992). An outbreak of multidrug-resistant tuberculosis among hospitalized patients with the acquired immunodeficiency syndrome. N Engl J Med 326 (23): 1514-1521.

Espinal MA, Kim SJ, Surarez PG (2000).Standard short-course chemotherapy for drug-resistant tuberculosis treatment outcome in 6 countries. JAMA, 283:2537-45.

Federal ministry of health (2008). Tuberculosis, leprosy and TB/HIV prevention and control programme manual, 4-5.

Federal Ministry of Health (2009). Guidelines for program and clinical management of Drug resistance Tuberculosis. Ethiopia, 3-4.

Ferrer G, Acuna-Villaorduna C, Escobedo M(2010). Outcomes of multidrug-resistant tuberculosis among binational cases in El Paso, Texas. Am J Trop Med Hyg; 83(5):1056-8.

Fischl MA, Uttamchandani RB, Daikos GL (1992). An outbreak of tuberculosis caused by multiple-drug resistant tubercle bacilli among patients with HIV infection. Ann Intern Med, 117: 177-183.

Gandhi, N.R., Moll, A., Sturm, A.W., Pawinski, R., Govender, T., Lalloo, U., Zeller, K., Andrews, J., Friedland, G (2006). Extensively drug-resistant tuberculosis as a cause of death in patients co-infected with tuberculosis and HIV in a rural area of South Africa. Lancet 368, 1575-1580.

Ghafoor A and Khan AA. 2014. Emperical Study about Fears and Misconceptions of Aids and Hepatitis among Medical Students. Malaysian Journal of Medical and Biological Research, 1, 25-34.

Glynn, J. R., Warndorff, D. K., Fine, P. E., Munthali, M. M., Sichone, W. \& Ponninghaus, J. M. (1998). Measurement and determinants of tuberculosis outcome in Karonga District, Malawi. Bulletin of the World Health Organization, 76, $295-305$.

Goble M, Iseman M D, Madsen L A (1993). Treatment of 171 patients with pulmonary tuberculosis resistant to isoniazid and rifampicin; N. Engl. J. Med. 328 527-532.

Hadhrami SA, Saaban AB, Ibrahim AB, Shahzad M and Ahmad I. 2014. Linear Active Control Algorithm to Synchronize a Nonlinear HIV / AIDS Dynamical System. Asian Journal of Applied Science and Engineering, 3, 96-113.

Haifeng Wang, MM, Hongsheng Lin, MM, and Gening Jiang, MD (2008).Pulmonary Resection in the Treatment ofMultidrugResistant Tuberculosis ARetrospective Study of 56 Cases. the Thoracic Surgery, Shanghai Pulmonary Hospital, Tongji University, Shanghai, China,2008,Ann Thorac Surg;86:1640 -5.

Hopewell, C. H. \& Chaisson, R. E. (2000). Tuberculosis and human immunodeficiency virus infection. In: Tuberculosis. A Comprehensive International Approach, Reichmann, L. B. \& Hershfield, E. S. (editors). New York \& Base]: MarcelDekker, pp. 525-552.

Iseman MD; Iseman, Michael D. (1993). Treatment of multidrug-resistant tuberculosis. N Engl J Med 329 (11): $784-791$.

Jacaban R F (1994). Multiple drug Resstant Tuberculosis; Clin. Infect. Dis. 19 1-10

Jeon DS, Shin DO, Park SK (2011). Treatment outcome and mortality among patients with multidrug-resistant tuberculosis in tuberculosis hospitals of the public sectors, 26(1):33-41.

Johnson J, Kagal A and Bharadwaj R (2003). Factors associated with drug resistance in pulmonary tuberculosis; Indian J. Chest Dis.Allied Sci. 45 105-109.

Juaeun N and Petms Nkamsse N. (2002). Impact of resistance to anti-tuberculosis drugs on treatment outcome using World Health Organization standard regimens. Transactions of the royal society of tropical medicine and hygiene, 96,429-433.

Kapp, C (2006). XDR tuberculosis spreads across South Africa. Lancet 369, 729.

Kassu D, Daniel A, Eshetu L, Mekdes G, Beniam F (2008).Drug susceptibility of Mycobacterium tuberculosis isolates from smear negative pulmonary tuberculosis patients, Addis Ababa, Ethiopia. Ethiop.J.Health Dev.22 (2):212-215.

Leimane V and Leimans J (2006). Integrated DOTS and DOTS plus programs. Euro surveillance, 11(3):29-33.

Lienhardt, C., Manneh, K., Bouchier, V., Lahai, G., Milligan, P. J. M. \& McAdam, K. P. W. J. (1998). Factors determining the outcome of tieatrnent oi'adult smear-positive tuberculosis cases in The Gambia. International Journal of Tuberculosis and Lung Disease, 2, 7 12-7 18.

Masanja, M., \& Msuya, J. (2014). Social Changes Imposed by HIV and AIDS in Rural Areas: An Empirical Evidence of Sex Education in Maswa District, Tanzania. Asian Journal Of Applied Science And Engineering, 3(8), 49-62. doi:10.15590/ajase/2014/v3i8/54484

Miguel G., Madariaga, MD, Umesh G. Lalloo, MBChB, MD, FCCP, Susan Swindells, MBBS (2008). Extensively Drug-resistant Tuberculosis. The American Journal of Medicine, 121, 835-844.

Mwinga A (2001). Drug resistant tuberculosis in Africa; Ann. N. Y. Acad. Sci. 953 106-112.

Neel R Gandhi, Paul Nunn, Keertan Dheda, H Simon Schaaf, Matteo Zignol, Dick van Soolingen, Paul Jensen, Jaime Bayona(2010).Multidrug-resistant and extensively drug-resistant tuberculosis a threat to global control of tuberculosis.Lancet, 375: 1830-43.

Pitchenik AE, Burr J, Laufer M (1990). Outbreaks of drug-resistant tuberculosis at AIDS centre. Lancet, 336: $440-441$.

Prasad R (2005). MDR TB Current Status; Indian J. Tuberc. 52 121-131

Sharma SK and Mohan A (2003). Scientific basis of directly observed treatment, short course (DOTS); J. Indian Med. Assoc. 101 $157-158,166$.

Sharma SK and Mohan A (2004). Multidrug-resistant tuberculosis; Indian J. Med. Res. 120 354-376.

Sharma SK and Mohan A (2006). Multidrug-resistant tuberculosis: a menace that threatens to destabilize tuberculosis control; Chest 130 261-272

St.Peter's TB Specialized Hospital (2007). Unpublished Human Resource Manual. 8:10-15.

Tadesse T, Hawaz Y and Zewdneh D. 2014. Patterns of Chest Radiographic Findings in Neonates with Respiratory Distress Admitted to the Neonatal Intensive Care Unit in Tikur Anbesa Specialized Hospital. Malaysian Journal of Medical and Biological Research, 1, 73-79. 
Tupasi T.E, Quelapio M.I.D, Orillaza R.B, Alcantara C, Mira N.R.C, Abeleda M.R, Belen V.T, Arnisto N.M, Rivera A.B, Grimaldo E, Derilo J.O, Dimarucut W, Arabit M, Urboda D (2003). DOTS-Plus for multidrug-resistant tuberculosis in the Philippines: global assistance urgently needed, Tropical Disease Foundation 2003.

Vaira L, Vija R, Timothy H , Evija Z, Vija Sk, Lorna E T, Kayla F L, Charles D (2005). Clinical outcome of individualized treatment of multidrug resistant tuberculosis in Latvia: a retrospective cohort study :Lancet 2005; 365: 318-26

WHO (2003). 7th annual report on global tuberculosis control. Geneva (2003).

World health organization (2010). Drug-resistant tuberculosis now at record levels.18, March.

World health organization (2010). Report on MDR-TB Nairobi Kenya 19, March.

www.cdc.gov/tb/publications/factsheets.

www.who.int/tb/challenges/mdr/

Zahan, N. (2013). HIV/AIDS Risk among the Children of Bangladesh. ABC Journal Of Advanced Research, 2(2), 30-43. Retrieved from http://journals.abc.us.org/index.php/abcjar/article/view/4.3\%20Revised 